DOI: $10.17516 / 1997-1397-2021-14-5-566-572$

УДК 512.5

\title{
On Reductants of Two Groups
}

\section{Dmitry P. Fedchenko* \\ Vitaly A. Stepanenko Rustam V. Bikmurzin Victoria V. Isaeva}

Siberian Federal University

Krasnoyarsk, Russian Federation

Received 04.02.2021, received in revised form 28.02.2021, accepted 06.03.2021

Abstract. In this paper we consider the reductant of the dihedral group $D_{n}$, consisting of a set of axial symmetries, and the sphere $S^{2}$ as a reductant of the group $\mathrm{SU}(2, \mathbb{C}) \cong S^{3}$ (the group of unit quaternions). By introducing the Sabinin's multiplication on the reductant of $D_{n}$, we get a quasigroup with unit.

Keywords: groups reductants, quasigroups

Citation: D.P. Fedchenko, V.A.Stepanenko, R.V. Bikmurzin, V.V. Isaeva, On Reductants of Two Groups, J. Sib. Fed. Univ. Math. Phys., 2021, 14(5), 566-572.

DOI: $10.17516 / 1997-1397-2021-14-5-566-572$.

\section{Introduction}

Nonassociative structures in modern algebra is not only mathematical curiosity but actively developed direction. The subtraction on a set of integers is not associative. Indeed, $3-(2-1)=2$, and $(3-2)-1=0$. On the set of real numbers we introduce the averaging operation $a * b=\frac{a+b}{2}$, then

$$
(a * b) * c=\frac{\frac{a+b}{2}+c}{2},
$$

and

$$
a *(b * c)=\frac{a+\frac{b+c}{2}}{2} .
$$

The vector product in three-dimensional space is nonassociative. Recall that

$$
a \times b=\left(a_{2} b_{3}-a_{3} b_{2}, a_{3} b_{1}-a_{1} b_{3}, a_{1} b_{2}-a_{2} b_{1}\right),
$$

where $a=\left(a_{1}, a_{2}, a_{3}\right)$ and $b=\left(b_{1}, b_{2}, b_{3}\right)$ are three-dimensional vectors.

Multiplication of octonionic imaginary units, see Tab. 1, does not have the property of associativity.

Therein

$$
\begin{aligned}
\imath^{2} & =J^{2}=\mathcal{J}^{2}=-1 \\
J z & =z^{*} J \\
h(q \mathcal{J}) & =(q h) \mathcal{J},(h \mathcal{J}) q=\left(h q^{*}\right) \mathcal{J},(h \mathcal{J})(q \mathcal{J})=-q^{*} h,
\end{aligned}
$$

*fdp@bk.ru

(C) Siberian Federal University. All rights reserved 
Table 1

\begin{tabular}{r|rrrrrrrr|} 
& 1 & $\imath$ & $J$ & $\imath J$ & $\mathcal{J}$ & $\imath \mathcal{J}$ & $J \mathcal{J}$ & $\imath J \mathcal{J}$ \\
\hline 1 & 1 & $\imath$ & $J$ & $\imath J$ & $\mathcal{J}$ & $\imath \mathcal{J}$ & $J \mathcal{J}$ & $\imath J \mathcal{J}$ \\
$\imath$ & $\imath$ & -1 & $\imath J$ & $-J$ & $\imath J$ & $-\mathcal{J}$ & $-\imath J \mathcal{J}$ & $J \mathcal{J}$ \\
$J$ & $J$ & $-\imath J$ & -1 & $\imath$ & $J \mathcal{J}$ & $\imath J \mathcal{J}$ & $-\mathcal{J}$ & $-\imath \mathcal{J}$ \\
$\imath J$ & $\imath J$ & $J$ & $-\imath$ & -1 & $\imath J \mathcal{J}$ & $-J \mathcal{J}$ & $\imath \mathcal{J}$ & $-\mathcal{J}$ \\
$\mathcal{J}$ & $\mathcal{J}$ & $-\imath \mathcal{J}$ & $-J \mathcal{J}$ & $-\imath J \mathcal{J}$ & -1 & $\imath$ & $J$ & $\imath J$ \\
$\imath \mathcal{J}$ & $\imath \mathcal{J}$ & $\mathcal{J}$ & $-\imath J \mathcal{J}$ & $J \mathcal{J}$ & $-\imath$ & -1 & $-\imath J$ & $J$ \\
$J \mathcal{J}$ & $J \mathcal{J}$ & $\imath J \mathcal{J}$ & $\mathcal{J}$ & $-\imath \mathcal{J}$ & $-J$ & $\imath J$ & -1 & $-\imath$ \\
$\imath J \mathcal{J}$ & $\imath J \mathcal{J}$ & $-J \mathcal{J}$ & $\imath \mathcal{J}$ & $\mathcal{J}$ & $-\imath J$ & $-J$ & $\imath$ & -1 \\
\hline
\end{tabular}

where $z$ is an arbitrary complex number, and $h, q$ are quaternions. Also $z^{*}$ and $q^{*}$ are conjugate quantities, see, for example, [1]. If we add a fourth imaginary unit $W$ with the property $W^{2}=-1$ and expand the system of relations

$$
\begin{aligned}
\imath^{2} & =J^{2}=\mathcal{J}^{2}=\Omega^{2}=-1 ; \\
J z & =z^{*} J \\
h(q \mathcal{J}) & =(q h) \mathcal{J},(h \mathcal{J}) q=\left(h q^{*}\right) \mathcal{J},(h \mathcal{J})(q \mathcal{J})=-q^{*} h ; \\
o(p W) & =(p o) W,(o W) p=\left(o p^{*}\right) W,(o W)(p W)=-p^{*} o ;
\end{aligned}
$$

then we get a set of sedenions, therein $o, p \in \mathbb{O}$. These are hexadecimal numbers. Thus we have a sequence of embeddings of hypercomplex number systems

$$
\mathbb{R} \subset \mathbb{C} \subset \mathbb{H} \subset \mathbb{O} \subset \mathbb{S} \subset \ldots,
$$

obtained one from the other using the Cayley-Dickson doubling procedure. Here $\mathbb{R}$ and $\mathbb{C}$ are fields of real and complex numbers, respectively, $\mathbb{H}$ is algebra (body in Russian) of quaternions, $\mathbb{O}$ is analytic Moufang loop of octonions, see for example [2], and $\mathbb{S}$ is loop of sedenions.

In Sections 1 and 2 we recall the basic concepts from the theory of quasigroups, and also point out the close relationship between quasigroups and finite automata. The application of FA in the theory of periodic groups was discussed in the article [3].

In Section 3 we discuss the reductant of the dihedral group $D_{n}$ consisting of axial symmetries. Thus, we illustrate the main ideas proposed in [4]. Following Sabinin we call by a reductant an arbitrary subset of a group. Very close questions are discussed in the paper [5], where twisted subsets of the dihedral group are considered. The authors of the paper call a subset $K$ of the group $G$ twisted if $e \in K$ and $x y^{-1} x \in K$ for all $x, y \in K$. An example of a twisted subset in $D_{n}$ is given by the involutions together with the unit.

In section 4 we show that the sphere $S^{2}$ is a reductant of the group $\mathrm{SU}(2, \mathbb{C})$.

\section{Quasigroups}

The set $Q$, considered together with some binary operation $*$, will be called a groupoid (or magma) $\langle Q, *\rangle$.

Definition 1. The groupoid $\langle Q, *\rangle$ is called a quasigroup if for any elements a and $b$ of the $Q$ equations

$$
a * x=b, y * a=b
$$

are always uniquely solvable. 
The Definition 1 is equivalent to the invertibility of the $*$ operation on the right and left, see, for example, [6, Chapter I]. In the finite-dimensional case this means that each row and column of the Cayley table of the groupoid $\langle Q, *\rangle$ are permutations of elements from $Q$. The quasigroup $\langle Q, *\rangle$ with a unit will be called a unital quasigroup or a loop. Note that in the definition of a quasigroup, the binary operation, in general, does not require associativity. In other words, a finite quasigroup is a nonassociative Latin square.

By a reductant $\mathcal{R}$ of the group $\langle\mathcal{G}, \circ\rangle$ we will call any subset of it. We introduce, quite naturally, the law of composition on $\mathcal{R}$, see, for example, [4].

$$
m_{1} * m_{2}=\operatorname{proj}\left(m_{1} \circ m_{2}\right),
$$

where $m_{1}$ and $m_{2}$ are arbitrary elements of $\mathcal{R}$, and proj: $\mathcal{G} \rightarrow \mathcal{R}$ is a projector on a reductant. We know this concept from the works of Sabinin.

In this paper we show that if we consider the set of axial symmetries in the dihedral group $D_{n}$ as $\mathcal{R}$, and arrange proj as

$$
\operatorname{proj}: r_{2 \pi k / n} \mapsto m_{\pi k / n},
$$

$k=0, \ldots, n-1$, then $\mathcal{R}$ will be endowed with the unital quasigroup structure. Here $r_{\varphi}$ is a counterclockwise rotation of $\varphi$, and $m_{\psi}$ is an axial symmetry relative to a straight line with an angle of inclination $\psi$.

\section{Quasigroup on a finite automaton}

Let $K_{d}$ be a complete graph, whose numbered vertices are represented by beads moving along the edges. The symbol 0 denotes an empty position where any of the beads can be moved. Fig. 1 shows the complete graph $K_{5}$.

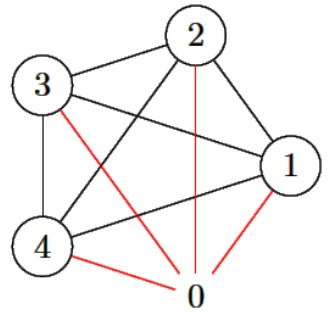

Fig. 1

Let $A=\left(Q, \mathcal{A}, \delta, q_{0}, F\right)$ be a deterministic finite automaton with the transition function $\delta: Q \times \mathcal{A} \rightarrow Q$, and $S=(Q, \mathcal{A}, \delta)$ be a semiatomaton see, for example, [7]. Consider a semiautomaton $S$ whose state set $Q$ coincides with the permutation group $S_{d}$ of vertices $\{1,2, \ldots, d-1,0\}$ of the graph $K_{d}$, and the input alphabet $\mathcal{A}$ is equal to the set $\{1,2, \ldots, d-1\}$ with $\delta:(s, j) \mapsto \sigma$, where the permutation $\sigma \in S_{d}$ is equal to $s$ up to the permutation of the elements $j$ and $0, j=1,2, \ldots, d-1$.

For $d=3$ we get the graph, which can be depicted in the way shown in Fig. 2.

Here $a, b, c, d, e$ and $f$ are elements of the symmetric group $S_{3}$, where $a=(1,2,0), b=(1,0,2)$, $c=(0,1,2), d=(2,1,0), e=(2,0,1), f=(0,2,1)$. It is easy to see that the set $S=\{a, c, e\}$ is a normal subgroup in $S_{3}$. On the set of involutions $\mathcal{R}=\{b, d, f\} \subset S_{3}$ we can introduce a quasigroup multiplication by the rule

$$
m_{i} * m_{j}=\operatorname{proj}\left(m_{i} m_{j}\right),
$$




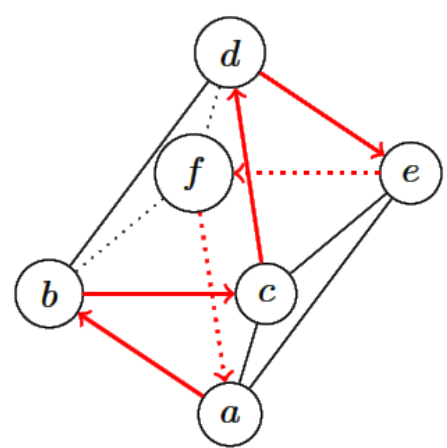

Fig. 2

where proj is the projection operator on $\mathcal{R}$ along the Hamiltonian cycle highlighted in red in Fig. 2, and $m_{i} m_{j}$ is the product in the group $S_{3}$. We get a nonassociative multiplication table (Tab. 2).

Table 2 .

\begin{tabular}{c|ccc}
$*$ & $b$ & $d$ & $f$ \\
\hline$b$ & $b$ & $d$ & $f$ \\
$d$ & $f$ & $b$ & $d$ \\
$f$ & $d$ & $f$ & $b$ \\
\hline
\end{tabular}

Indeed, different ways of placing parentheses lead to different results $(f d) b=d$, and $f(d b)=b$. We have obtained a third-order quasigroup.

\section{Reductant of a dihedral group}

Consider a regular $n$-gon. The group of its symmetries (dihedral group) consists of a subgroup of rotations $S=\left\{r_{2 \pi k / n}\right\}$ and axial symmetries $\mathcal{R}=\left\{m_{\pi k / n}\right\}, k=0,1, \ldots, n-1$ which are represented by matrices

$$
\left(\begin{array}{rr}
\cos 2 \pi k / n & -\sin 2 \pi k / n \\
\sin 2 \pi k / n & \cos 2 \pi k / n
\end{array}\right)
$$

and

$$
\left(\begin{array}{rr}
\cos 2 \pi k / n & \sin 2 \pi k / n \\
\sin 2 \pi k / n & -\cos 2 \pi k / n
\end{array}\right)
$$

respectively. It is easy to see that the projector proj: $S \rightarrow \mathcal{R}$ just throws the minus sign in the second column from the first row to the second. More strictly, the projection is carried out by multiplying the rotation matrix by the Pauli matrix on the right:

$$
\begin{aligned}
\operatorname{proj}\left(r_{2 \pi k / n}\right) & =\left(\begin{array}{rr}
\cos 2 \pi k / n & -\sin 2 \pi k / n \\
\sin 2 \pi k / n & \cos 2 \pi k / n
\end{array}\right)\left(\begin{array}{rr}
1 & 0 \\
0 & -1
\end{array}\right) \\
& =\left(\begin{array}{rr}
\cos 2 \pi k / n & \sin 2 \pi k / n \\
\sin 2 \pi k / n & -\cos 2 \pi k / n
\end{array}\right) .
\end{aligned}
$$


Obviously, $\operatorname{proj}^{2}=\mathrm{id}$, where id is an identical transformation. Multiplying the matrices of two axial symmetries, we get

$$
\begin{array}{r}
\left(\begin{array}{rr}
\cos 2 \pi k / n & \sin 2 \pi k / n \\
\sin 2 \pi k / n & -\cos 2 \pi k / n
\end{array}\right)\left(\begin{array}{rr}
\cos 2 \pi l / n & \sin 2 \pi l / n \\
\sin 2 \pi l / n & -\cos 2 \pi l / n
\end{array}\right) \\
=\left(\begin{array}{cc}
\cos 2 \pi(k-l) / n & -\sin 2 \pi(k-l) / n \\
\sin 2 \pi(k-l) / n & \cos 2 \pi(k-l) / n
\end{array}\right) .
\end{array}
$$

Let $0<\alpha<\pi$, then $m_{\alpha}=m_{\pi+\alpha}$ and $m_{-\alpha}=m_{\pi-\alpha}$. If $k-l \geqslant 0$, then

$$
m_{\pi k / n} * m_{\pi l / n}=m_{\pi(k-l) / n}
$$

if $k-l<0$, then

$$
m_{\pi k / n} * m_{\pi l / n}=m_{\pi(n+k-l) / n} .
$$

Let's write out the multiplication table of the axial symmetries of the dihedral group $D_{n}$ (Tab. 3). For more elegance, instead of $m_{\pi k / n}, k \in \mathbb{Z} \cap[-n+1, n-1]$ we will simply write the $k$.

Table 3.

\begin{tabular}{c|rrrrrrrr|}
$*$ & 0 & 1 & 2 & 3 & 4 & 5 & $\ldots$ & $n-1$ \\
\hline 0 & 0 & $n-1$ & $n-2$ & $n-3$ & $n-4$ & $n-5$ & $\ldots$ & 1 \\
1 & 1 & 0 & $n-1$ & $n-2$ & $n-3$ & $n-4$ & $\ldots$ & 2 \\
2 & 2 & 1 & 0 & $n-1$ & $n-2$ & $n-3$ & $\ldots$ & 3 \\
3 & 3 & 2 & 1 & 0 & $n-1$ & $n-2$ & $\ldots$ & 4 \\
4 & 4 & 3 & 2 & 1 & 0 & $n-1$ & $\ldots$ & 5 \\
5 & 5 & 4 & 3 & 2 & 1 & 0 & $\ldots$ & 6 \\
$\vdots$ & $\vdots$ & $\vdots$ & $\vdots$ & $\vdots$ & $\vdots$ & $\vdots$ & $\ldots$ & $\vdots$ \\
$n-1$ & $n-1$ & $n-2$ & $n-3$ & $n-4$ & $n-5$ & $n-6$ & $\ldots$ & 0 \\
\hline
\end{tabular}

\section{4. $S^{2}$ sphere}

Calculate the product of the points $w=\left(w_{1}, w_{2}, w_{3}\right)$ and $v=\left(v_{1}, v_{2}, v_{3}\right)$ from sphere $S^{2} \subset$ $\mathrm{SU}(2, \mathbb{C})$ represented by complex matrices

$$
\begin{aligned}
& \left(\begin{array}{ll}
\imath w_{1} & -w_{2}-\imath w_{3} \\
w_{2}-\imath w_{3} & -\imath w_{1}
\end{array}\right)\left(\begin{array}{ll}
\imath v_{1} & -v_{2}-\imath v_{3} \\
v_{2}-\imath v_{3} & -\imath v_{1}
\end{array}\right) \\
& =\left(\begin{array}{cc}
-\langle w, v\rangle+\imath\left(w_{2} v_{3}-w_{3} v_{2}\right) & -\left(w_{3} v_{1}-w_{1} v_{3}+\imath\left(w_{1} v_{2}-w_{2} v_{1}\right)\right) \\
w_{3} v_{1}-w_{1} v_{3}-\imath\left(w_{1} v_{2}-w_{2} v_{1}\right) & -\langle w, v\rangle-\imath\left(w_{2} v_{3}-w_{3} v_{2}\right)
\end{array}\right) \\
& =\left(\begin{array}{cc}
\xi_{0}+\imath \xi_{1} & -\left(\xi_{2}+\imath \xi_{3}\right) \\
\xi_{2}-\imath \xi_{3} & \xi_{0}-\imath \xi_{1}
\end{array}\right) \\
& =\xi
\end{aligned}
$$

where the norm of a vector $\left.\|\left(\xi_{0}, \xi_{1}, \xi_{2}, \xi_{3}\right)\right) \|_{\mathbb{R}^{4}}$ coincides with the determinant of the matrix $\xi$ and is equal to one $(\|\xi\|=\operatorname{det} \xi=1)$. 
Now we project $\xi \in \mathrm{SU}(2, \mathbb{C})$ back onto the reductant. To do this, we will twist the $\xi$ as follows:

$$
\begin{aligned}
& \left(\begin{array}{ll}
a+\imath b & 0 \\
0 & a-\imath b
\end{array}\right)\left(\begin{array}{cc}
\xi_{0}+\imath \xi_{1} & -\left(\xi_{2}+\imath \xi_{3}\right) \\
\xi_{2}-\imath \xi_{3} & \xi_{0}-\imath \xi_{1}
\end{array}\right) \\
& =\left(\begin{array}{cc}
a \xi_{0}-b \xi_{1}+\imath\left(a \xi_{1}+b \xi_{0}\right) & -\left(a \xi_{2}-b \xi_{3}+\imath\left(a \xi_{3}+b \xi_{2}\right)\right) \\
a \xi_{2}-b \xi_{3}-\imath\left(a \xi_{3}+b \xi_{2}\right) & a \xi_{0}-b \xi_{1}+\imath\left(a \xi_{1}+b \xi_{0}\right)
\end{array}\right) \\
& =\left(\begin{array}{cc}
\eta_{0}+\imath \eta_{1} & -\left(\eta_{2}+\imath \eta_{3}\right) \\
\eta_{2}-\imath \eta_{3} & \eta_{0}-\imath \eta_{1}
\end{array}\right),
\end{aligned}
$$

where $a^{2}+b^{2}=1$. We are looking for $a$ and $b$ such that $\eta_{0}=0\left(a \xi_{0}=b \xi_{1}\right)$ and

$$
\begin{aligned}
1 & =\eta_{1}^{2}+\eta_{2}^{2}+\eta_{3}^{2} \\
& =\left(a \xi_{1}+b \xi_{0}\right)^{2}+\left(a \xi_{2}-b \xi_{3}\right)^{2}+\left(a \xi_{3}+b \xi_{2}\right)^{2} \\
& =a^{2}\left(1-\xi_{0}^{2}\right)+b^{2}\left(1+\xi_{1}^{2}\right) .
\end{aligned}
$$

Some location configurations of an arbitrary ellipse and circle $a^{2}+b^{2}=1$ are shown in Fig. 3 .
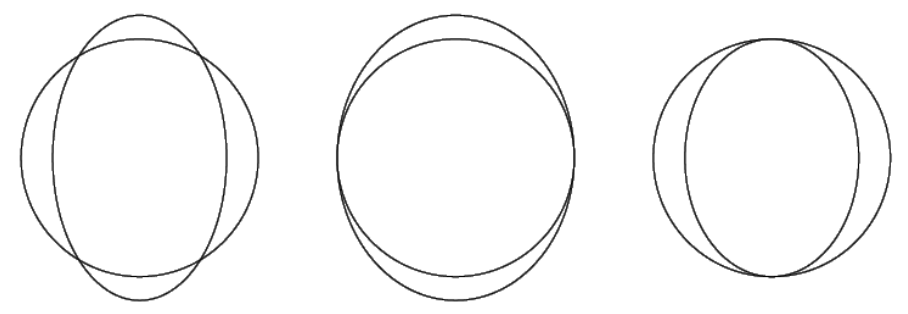

Fig. 3

The solution of the system

$$
\left\{\begin{array}{l}
a^{2}\left(1-\xi_{0}^{2}\right)+b^{2}\left(1+\xi_{1}^{2}\right)=1 \\
a^{2}+b^{2}=1
\end{array}\right.
$$

have the form

$$
\begin{aligned}
& b=-\sqrt{1-a^{2}}, \quad a^{2}-1 \neq 0, \quad \xi_{1}=-\frac{a \xi_{0}}{\sqrt{1-a^{2}}} \\
& b=-\sqrt{1-a^{2}}, \quad a^{2}-1 \neq 0, \quad \xi_{1}=\frac{a \xi_{0}}{\sqrt{1-a^{2}}} ; \\
& b=\sqrt{1-a^{2}}, \quad a^{2}-1 \neq 0, \quad \xi_{1}=-\frac{a \xi_{0}}{\sqrt{1-a^{2}}} ; \\
& b=\sqrt{1-a^{2}}, \quad a^{2}-1 \neq 0, \quad \xi_{1}=\frac{a \xi_{0}}{\sqrt{1-a^{2}}} \\
& a=-1, \quad b=0, \quad \xi_{0}=0 \\
& a=1, \quad b=0, \quad \xi_{0}=0 .
\end{aligned}
$$


This work was supported by the Krasnoyarsk Mathematical Center and financed by the Ministry of Science and Higher Education of the Russian Federation in the framework of the establishment and development of regional Centers for Mathematics Research and Education (Agreement No. 075-02-2020-1631).

\section{References}

[1] N.Jacobson, Lie algebras, Courier Corporation, 1979.

[2] A.I.Maltsev, Analytic loops, Matematiceskij sbornik, 78(1955), no. 3, 569-576 (in Russian).

[3] S.V.Aleshin, Finite automata and the Burnside problem for periodic groups, Math. Notes, 11(1972), 199-203. DOI: 10.1007/BF01098526

[4] L.V.Sabinin, Loop geometries, Mathematical notes of the Academy of Sciences of the USSR, 12(1972), no. 5, 799-805. DOI: 10.1007/BF01099069

[5] A.L.Myl'nikov Minimal non-group-like twisted subsets with involutions, Siberian Mathematical Journal, 48(2007),no. 5, 879-883. DOI: 10.1007/s11202-007-0090-5

[6] V.D.Belousov Foundations of the theory of quasigroups and loops, Nauka, Moscow, 1967 (in Russian).

[7] A.Ginzburg, Algebraic theory of automata, Academic Press, 2014.

\section{О редуктантах двух групп}

Дмитрий П. Федченко Виталий А. Степаненко Рустам В. Бикмурзин Виктория В. Исаева

Аннотация. В работе рассматриваются редуктант диэдральной группы $D_{n}$, состоящий из множества осевых симметрий, и сфера $S^{2}$ как редуктант группы $\mathrm{SU}(2, \mathbb{C}$ ) (группы единичных кватернионов). Введя сабининское умножение на редуктанте из $D_{n}$, мы получим квазигруппу с единицей.

Ключевые слова: редуктанты групп, квазигруппы. 